\title{
DIE IDENTIFIZIERUNG UND REPRÄSENTIERUNG DES QUERCUS-POLLEN IN EINIGEN SPANISCHEN ORANGENHONIGEN
}

\author{
Magda O. WEBER \\ Institut des Sciences de l'évolution, Laboratoire de Palynologie \\ Place Eugène-Bataillon, 34060 Montpellier Cedex
}

\section{ZUSAMMENFASSUNG}

Pollenmorphologische Untersuchungen bei 11 Orangenhonigen von der Ostküstenregion Spaniens wurden durchgeführt. Ein unbekannter, tricolporater Pollen, welcher als Leitpollen häufig an Stelle von Citrus-Pollen auftritt, wurde identifiziert. Die L.M. und R.E.M. Untersuchungen bei Honigsedimenten und frischen Referenzpollen zeigten, dass der unbekannte Pollen, ein anemophiler Pollen, der Familie Fagaceae, respektive der Gattung Quercus angehört. Zwei Arten, Quercus ilex L. und Q. coccifera L., sind in Honigen identifiziert worden. $Q$. coccifera-Pollen waren nur wenig vorhanden. Die qualitativen Analysen der Honigproben ergaben, dass von den 11 "Orangenhonigen" nur 3 die Norm erfüllten, um sie als Sortenhonige bzw. Orangenhonige taxieren zu können. In allen restlichen Proben dominierten die $Q$. ilexPollen über den Citrus-Pollen. Das ausgeprägte Orangenblütenaroma dieser Honige zeigte jedoch, dass sie beträchtliche Mengen an Citrus-Nektar aufweisen.

Die Ergebnisse dieses Berichtes zeigen den Zusammenhang zwischen dem Prozentsatz an Citrus- und Quercus-Pollen. Das Vorkommen und die Dominanz der Quercus ilex-Pollen in allen Honigen wird hier erklärt.

Das Pollenbild des Honigsedimentes ist in Tabelle I zusammengefasst.

Die pollenmorphologischen Ergebnisse der untersuchten Quercus-Arten der Ostküstenregion Spaniens: Quercus ilex L., $Q$. coccifera L., $Q$. suber L. sind beschrieben worden. Ein Bestimmungsschlüssel wurde zusätzlich angefertigt.

\section{EINLEITUNG}

In der Melissopalynologie wird ein Sortenhonig als ein Honig aus einseitiger Tracht definiert, in dessen Pollenbild ein Leitpollen mit einem Anteil von über $45 \%$ vorhanden ist. Auf Grund ihres absoluten Gehaltes an Pollenkörnern/Honigeinheit $(10 \mathrm{~g})$, werden die Sortenhonige in 3 Gruppen aufgeteilt (HAMmer, 1948, Demianowicz, 1961 und MaUrizio, 1949, 1955, 1958). Pollenarme : Lavandula, Robinia, Tilia; pollenreiche : Castanea, Myosotis; Honigsorten mit mittlerem Pollengehalt : Erica 
carnea, Rubus, Salix u.s.w. Orangenblütenhonige gehören in die Gruppe der pollenarmen Honigsorten (Maurizio, 1958). Die Blüten der Citrus-Arten sondern grosse Mengen Nektar ab, der 13-30\% Zucker enthält. Gewisse Orangensorten (Navel) besitzen sterile Antheren und produzieren keine oder missgebildete Pollen. Es wird deshalb angenommen, dass Orangenblütenhonige pollenarm sind. Der Gehalt an Orangennektar ist jedoch beträchtlich. Ein Anteil der Citrus-Pollen von $10 \% \mathrm{im}$ Honig genügt noch, um ihn als Sortenhonig zu deklarieren. Der prozentuelle Anteil der CitrusLeitpollen kann je nach Herkunft des Honigs verschieden sein. Im kalifornischen Honig kommt der Citrus-Pollen als Leitpollen mit einem Anteil von 46-65 \% vor (MAURIzIo, 1958), kann aber auch nur als Begleitpollen mit 14-40\% auftreten.

Bei den Routineuntersuchungen der spanischen Orangenhonige wurde öfter beobachtet, dass an Stelle von Citrus-Pollen ein unbekannter, tricolporater Pollen als Leitpollen auftritt. Im Jahre 1975 erhielt ich 11 Proben von Dr. A. Maurizio (Liebefeld), um diesen Pollen zu identifizieren. Das Material wurde weiter für die qualitativen Analysen des Honigsedimentes verwendet, um den Prozentsatz an Citrusund Quercus-Pollen feststellen zu können.

\section{MATERIAL UND METHODE}

Zwecks Identifizierung eines unbekannten Pollen wurden 11 spanische Orangenhonige untersucht. Zwei stammten aus Valencia (Probe I u. II), einer aus Castellon (Probe VII) und einer aus Nules (Probe IX), der Rest waren Handelshonige ohne Angaben über den Ort der Herkunft. Die Proben wurden zuerst "orientierend" analysiert, d.h. die häufig auftretenden Pollen wurden mit Referenzpollen nach morphologischen Merkmalen verglichen. Im Jahre 1976 (23-3/16-4) unternahm ich eine Reise nach Spanien, um die Vegetation und die Verbreitung der Bienenstöcke in den Plantagen der Ostküstenregion Spaniens kennenzulernen. Die Blüten der verschiedenen Citrus-Arten wie Citrus medica L., C. aurantium var. verna und var. dulcis wurden beobachtet. Ein Herbarmaterial (ca. 200 Pflanzenarten) aus der Umgebung wurde gesammelt. Besonderer Wert wurde auf die sichere Bestimmung des Materials gelegt. Für die mikroskopische Untersuchung wurden die Pollen von Herbar- und Frischmaterial nach der Methode von ERdTMAN (1960) und MaURizio aufbereitet. Zur Feststellung der Variationsbreite der Pollenkörner wurden nach Möglichkeit verschiedene Herkünfte ein und derselben Art gemessen.

Die Pollengrösse wurde an acetolysierten und nicht behandelten Pollen gemessen.

Durchschnittswerte wurden von ca. 25 Pollen pro Präparat berechnet. Die Untersuchung der Skulpturelemente erfolgte unter der Ölimmersion. Für die genaue Identifizierung bis zur Art wurden R.E.M.-Aufnahmen nach der Methode des Geologischen Institutes der Universität Bern gemacht. Die pollenmorphologische Terminologie wird nach den Vorschlägen von ERDTMAN, 1969 und WoodHousE, 1959 gebraucht. Die Anfertigung der Honigpräparate und die Auswertung erfolgten nach den Angaben der Internationalen Kommission für Bienenbotanik (Louveaux, MAUrizıo, VoRwOHL, 1970). Einige Honigproben wurden nach meiner Methode acetolysiert, um die unterschiedliche Auswirkung der zwei Methoden feststellen zu können.

Nach dieser Präparation kann man die Pollen aus dem Honigsediment leichter mit den Referenzpollen der nicht-melissopalynologischen Literature vergleichen. Alle Untersuchungen, Präparationen und Photos wurden vom Autor durchgeführt. Das gesamte Material ist beim Autor archiviert. 
Den fest kandierten Honig im Wasserbad $\left(40^{\circ}\right) 10$ Min. stehen lassen. (3 Min.).

1. ca $20 \mathrm{~g}$ Honig in Zentrifugengläser geben, mit destilliertem $\mathrm{H}_{2} \mathrm{O}$ auffïllen und zweimal zentrifugieren

2. Das sedimentierte Material mit destilliertem $\mathrm{H}_{2} \mathrm{O}$ versetzen und danach einige Tropfen $10 \%$-iger KOH Lösung zugeben. Fur ca. 5 Min. im Wasserbad unter ständigem Rühren kochen lassen.

3. Material durch Porzellansieb sieben.

4. $1 \times$ zentrifugieren (ca. 2 Min.).

5. $1 \times$ waschen mit destilliertem $\mathrm{H}_{2} \mathrm{O}$, wieder zentrifugieren, Überstand abgiessen.

6. Zugabe von Eisessig (Entwässern), zentrifugieren, Überstand in Säureabguss abgiessen.

7. Acetolyse nach ERDTMan.

8. Der Probe wird 2-5 ml Glyzerin zugefügt und im Wasserbad (ca. $75^{\circ}$ ), 10 Min. gekocht. Warm zentrifugieren (10 Min.), Glyzerin abgiessen. Präparatherstellung.

\section{ERGEBNISSE}

Die Honig-Analysen erfolgten zuerst " orientierend ", d.h. die häufig auftretenden Pollen wurden zwecks Identifizierung mit Referenzpollen verglichen. Nach eingehenden Untersuchungen der Familien Rhamnaceae, Rutaceae, Vitaceae und Fagaceae, zeigte sich, dass der zu identifizierende Pollen der Familie Fagaceae, und zwar der Gattung Quercus angehört. Es wurden dabei nur die in der Ostküsten-Region Spaniens vorkommenden Quercus-Arten : Quercus ilex L., Q. coccifera L., $Q$. suber L., berücksichtigt. Eine Differentialdiagnose der verschiedenen Quercus-Arten wurde mit Hilfe des R.E.M. erreicht. Es ergab sich, dass der gesuchte Pollen der Art $Q$. ilex angehört. Gleichzeitig stellte sich heraus, dass im Honig noch $Q$. coccifera vorhanden war. Die Ergebnisse der qualitativen Analysen sind in Tabelle I zusammengefasst.

In allen 11 Honigproben dominierten die Quercus ilex-Pollen über die CitrusPollen. Nur 3 Honigproben könnten nach der Norm als Orangenhonige taxiert werden.

Im Folgenden werden die pollenmorphologischen Ergebnisse beschrieben.

\section{BESPRECHUNG DER POLLENMORPHOLOGISCHEN ERGEBNISSE}

\section{Allgemeine Pollenbeschaffenheit}

Die Pollenkörner sind klein bis mittelgross, tricolporate oder tricolpate und haben in der Regel drei sich gleichende Aperturen. Die 3 tief eingeschnittenen Colpi sind deutlich mit breiten Lippen ausgebildet, die eine feine, glatte Membran zeigen. Porus, 
wenn vorhanden, klein, ungeformt und ohne erkennbare Struktur, ist aber nicht bei allen Quercus-Arten ausgebildet. Exine : mitteldick, zweischichtig, fein granulat. Die Pollen haben eine senfgelbe bis olivgrüne Farbe.

Quercus ilex L. (Tafel I, II, IV). Frisches Pollenmaterial : Forêt du Parc, Pézenas; Colombier (S. Frankreich); Llorett; Tibidabo (Spanien).

Tricolporate Pollen, klein bis mittelgross, $\mathrm{P}: 20,8-25,5$, E. : 23,6-28,8, P/E : 0,88 suboblat. Der Abstand im Intercolpien $t=6 \mu$. Polansicht : Umriss dreieckig, gewölbt mit tief eingeschnittenen Colpi, welche nicht bis zum Pol reichen.
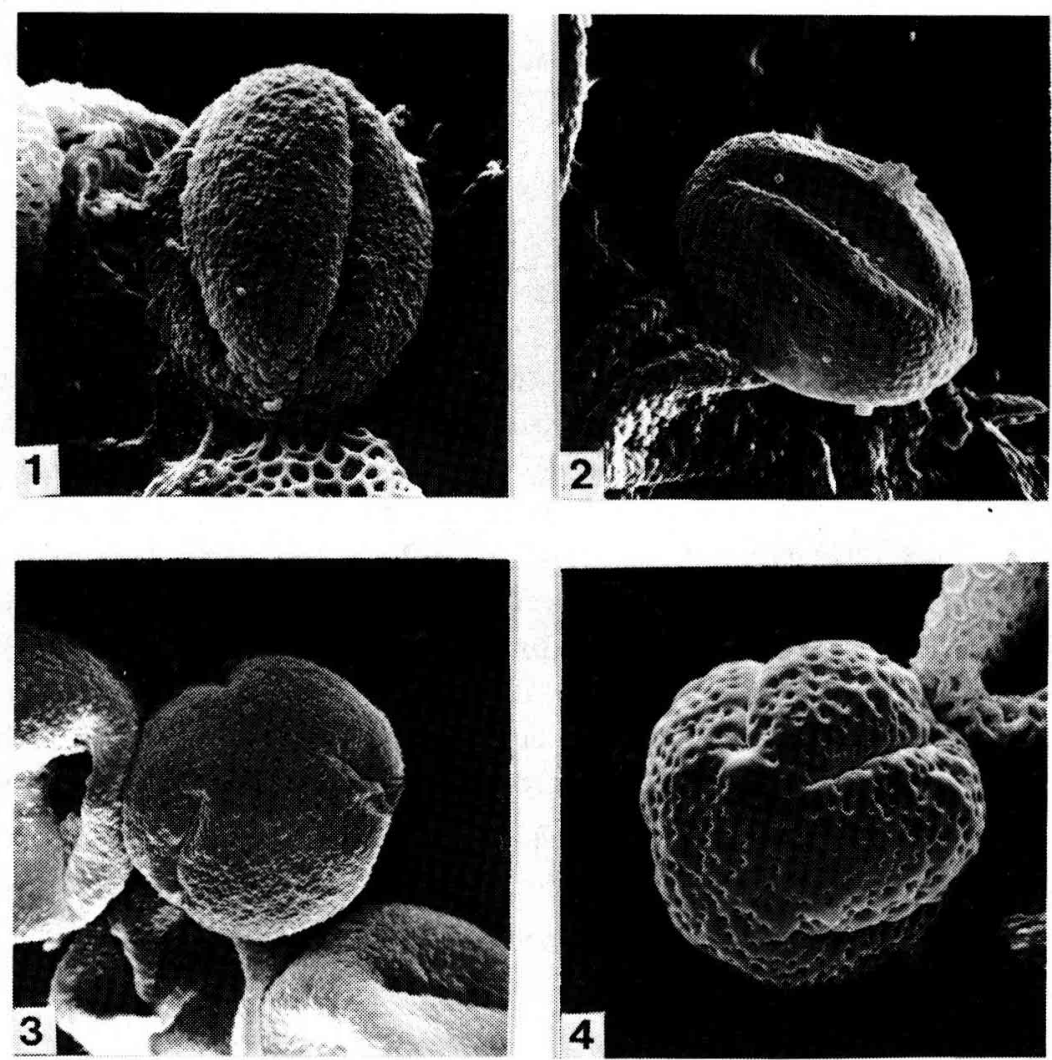

TAFEL I. - Referenz-Pollen im R.E.M.

FIG. I. - Reference pollens in S.E.M.

1. - Quercus ilex L., $1560 \times$.

2. - Quercus coccifera L., $1760 \times$.

3. - Quercus suber L., $1170 \times$.

4. - Citrus aurantium var. dulcis, $1500 \times$. 


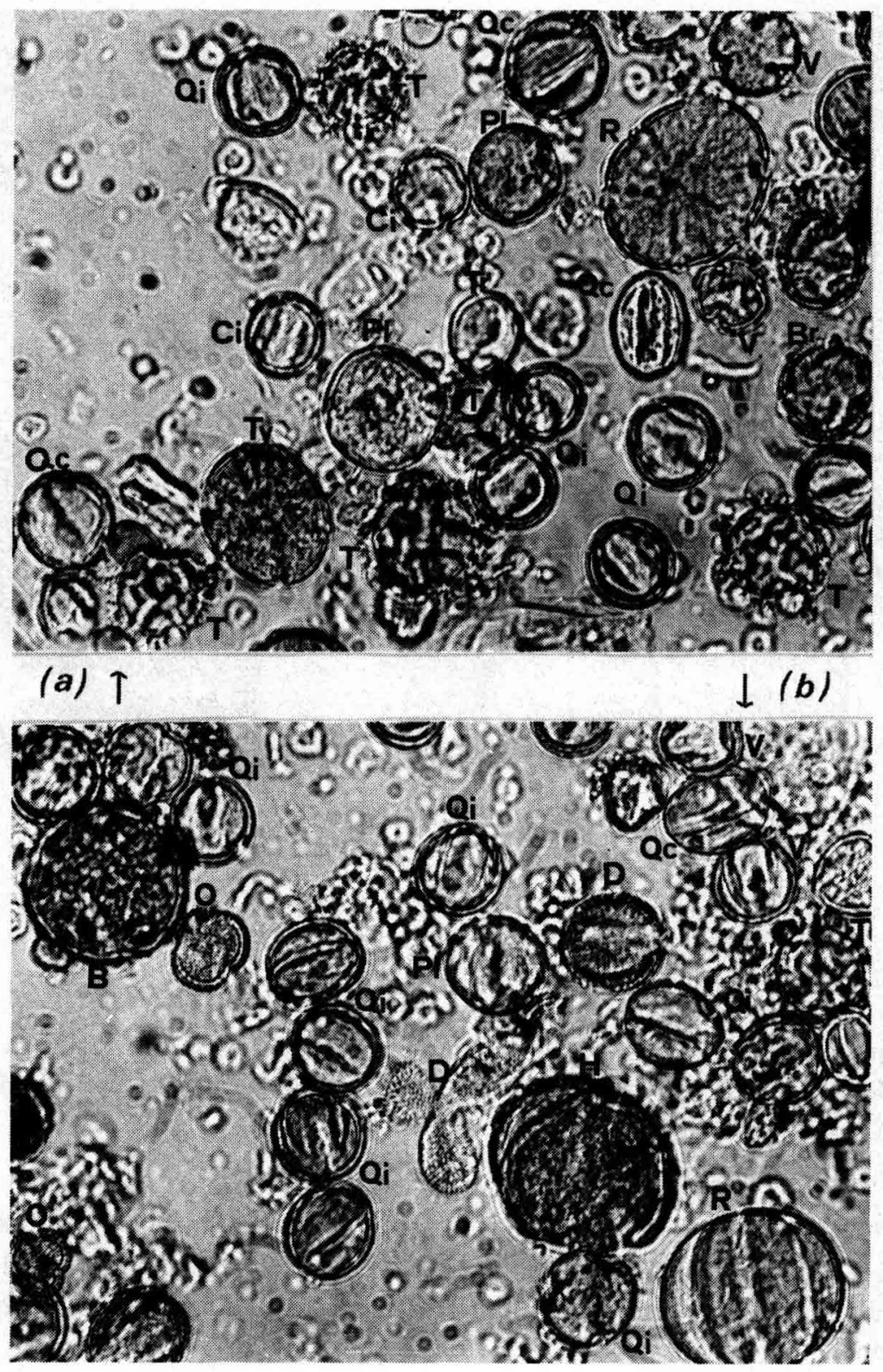

TAFEL II. - Das Pollenspektrum in einigen spanischen Orangenhonigen.

FIG. II. - Pollen spectrum in some spanish orange honeys.

(a) Olea europea (O), Vitis sp. (V), Brassica sp. (B), Rosmarinus off. (R), Quercus coccifera-Gruppe (Qc), Quercus ilex-Gruppe (Qi), Plantago sp.(S), Thymus sp. (Ty), Citrus sp. (Ci), Compositae T-Form (T).

(b) Borago off. (B), Diplotaxis erucoides (D), Helianthemum sp. (H). 


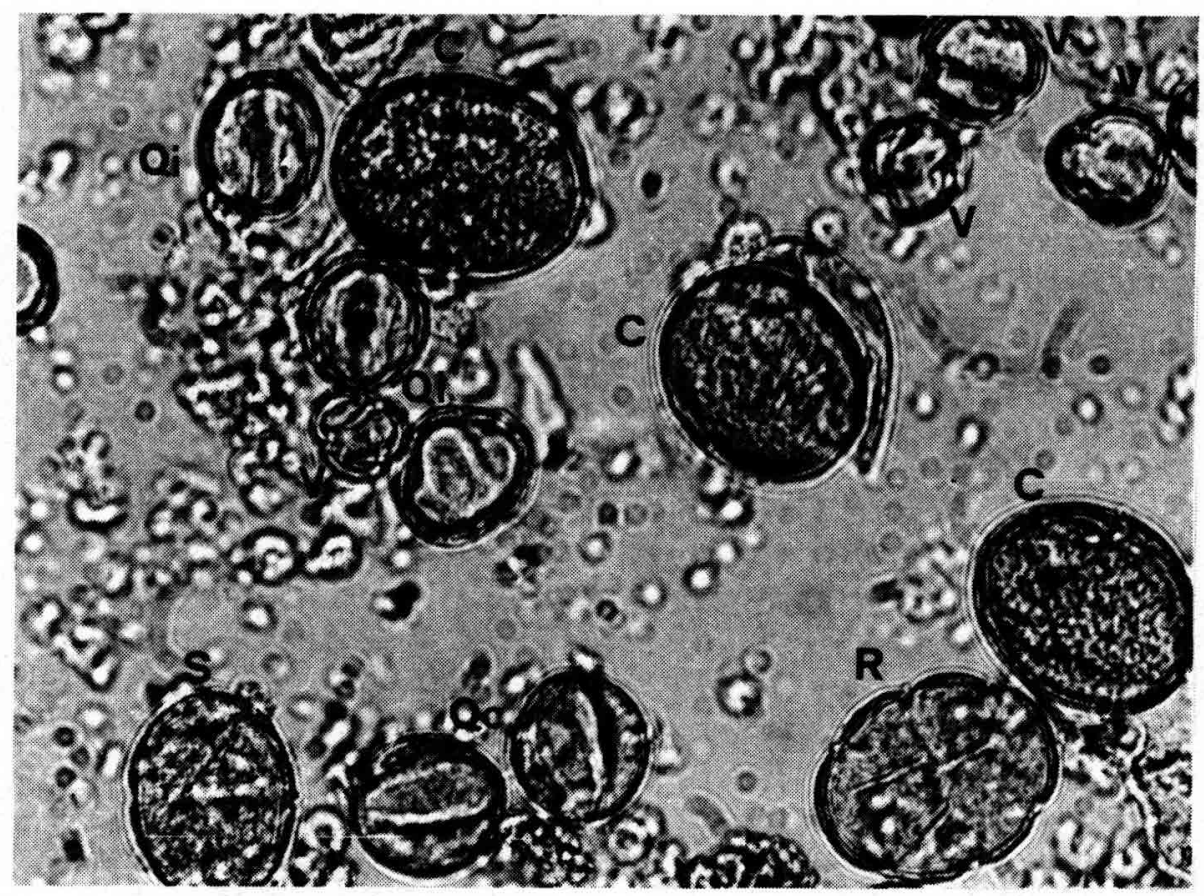

TAFEL III. - Das Pollenspektrum in einigen spanischen Orangenhonigen.

FIG. III. - Pollen spectrum in some spanish orange honeys.

Cistus sp. (c), Salvia sp. (S.).

Äquatoransicht : Umriss elliptisch. Ausbuchtungen stark konvex. Jeder Colpus hat in der Mitte einen Porus, welcher bei starker Öffnung kreisförmig ist und nur bei halber Öffnung eine elliptische Form zeigt.

Struktur und Skulpturelemente der Exine :

Exine ca. 2,3 $\mu$ dick. Ektexine dicker als Endexine. Im L.M. erscheint sie granulat.

Im R.E.M. mit unregelmässigen verrucaten Skulpturelementen welche bis an den Pol erhalten sind. Die Lippen der Colpi \pm breit, zeigen eine feine, granulate bis glatte Membran.

Quercus coccifera L. (Tafel I, II, IV) Frisches Pollenmaterial : Spanisch Marokko, W. Ceuta; Provence; Montpellier (S. Frankreich); Valencia (Spanien). Tricolporate, tricolpate Pollen, relativ klein, P : 20,8-24,7, E : 18,3-21,3, P/E 1,15 subprolat. Der Abstand im Intercolpien $\mathrm{t}=5 \mu$. Polansicht : Umriss dreieckig. Colpi sind locker und weiter entfernt vom Pol als bei $Q$. ilex, so dass der dreieckige Umriss fast rundlich erscheint. Aquatoransicht : Umriss elliptisch. 

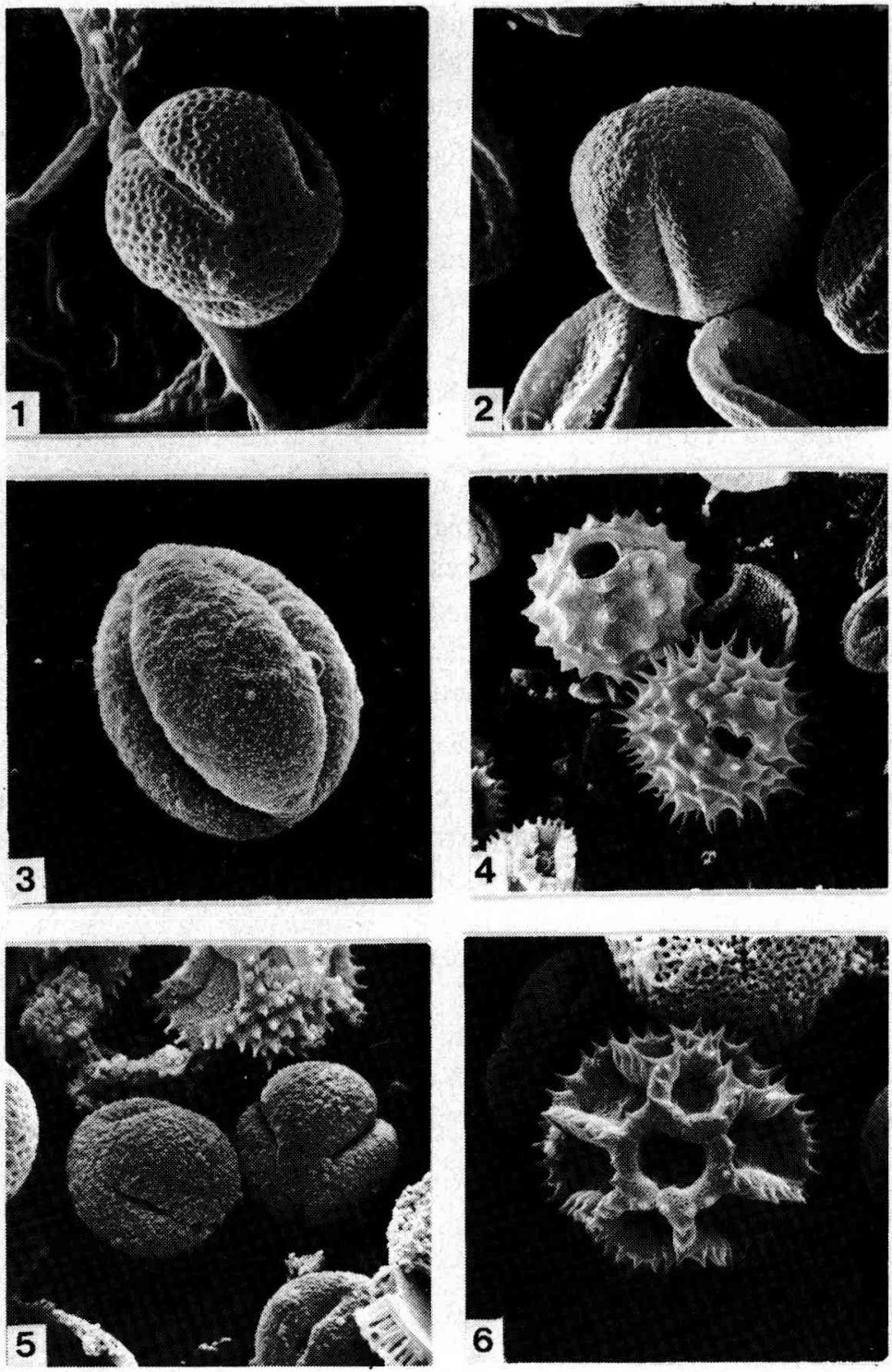

TAFEL IV. - Das Pollenbild der spanischen Orangenhonig im R.E.M.

FIG. IV. - Pollen of Spanish orange honeys in S.E.M.

1. - Citrus aurantium L., $1560 \times$.

2. - Vitis vinifera $\mathrm{L} ; 1760 \times$.

3. - Quercus coccifera Gruppe, $900 \times$.

4. - Chrysanthemum coronarium L. (oben upper) und Calendula arvensis L. (unten lower), $780 \times$.

5. - Quercus ilex-Gruppe, $1200 \times$.

6. - Taraxacum officinale, $1200 \times$. 
Ausbuchtungen im spitzen Winkel unterbrochen. Die Lippen der Colpi stehen weit auseinander und haben eine glatte Membran. Der kleine ungeformte Porus ist schwer $\mathrm{zu}$ erkennen und nicht immer vorhanden.

Struktur und Skulpturelemente der Exine :

Exine ca. 2,8 $\mu$ dick. Ektexine gleich dick wie die Endexine.

Im L.M. erscheint die Exine fein granulat bis glatt. Im R.E.M. mit lockeren, verrucaten Skulpturelementen, welche feine, unregelmässig verteilte Mikroperforationen aufweisen.

Quercus suber L. (Tafel I), Frisches Pollenmaterial : Lorett; Figueras; Barcelona (Spanien); Montpellier; Provence (S. Frankreich), Herbar Sutter, Bot. Inst. Bern.

Tricolporate, häufig tricolpate Pollen, mittelgross $\mathrm{P}: 35,2-36-2, \mathrm{E}: 27,6-32,2, \mathrm{P} / \mathrm{E}$ 1,09 sphäroid. Der Abstand im Intercolpien $t=8 \mu$. Polansicht : Umriss rund, dreieckig. Die 3 tief eingeschnittenen Colpi reichen nicht bis zum Pol. Aquatoransicht : Umriss elliptisch, kreisförmig. Ausbuchtungen verlaufen gleichmässig über den Äquator. Der kleine Porus ist nicht immer vorhanden. Die Lippen der Colpi sind mittelbreit und zeigen im R.E.M. eine feine, fast glatte Membran.

Struktur und Skulpturelemente der Exine :

Exine ca. $2 \mu$ dick. Ektexine gleich dick wie Endexine. Im L.M. erscheint sie fein granulat. Im R.E.M. mit gleich verteilten lockeren, verrucaten Skulpturelementen, welche dichte Mikroperforationen aufweisen. Sie sind bis an den Pol erhalten.

\section{BESTIMMUNGSSCHLÜSSEL}

Tricolporate Pollen $<30 \mu$, suboblat,

Exine unregelmässig verrucat ca. $2,3 \mu$

$\mathrm{t}=6 \mu$, Porus kreisförmig oder elliptisch $\ldots \ldots \ldots \ldots \ldots$ Quercus ilex $\mathrm{L}$.

Tricolporate, tricolpate Pollen bis

$25 \mu$, subprolat, Exine fein verrucat

ca. $2,8 \mu, \mathrm{t}=5 \mu$, Porus ungeformt $\ldots \ldots \ldots \ldots \ldots$ Quercus coccifera $\mathrm{L}$.

Tricolporate, tricolpate Pollen $>30 \mu$

sphäroid, Exine regelmässig

verrucat bis fein granulat, ca. $2 \mu$,

$\mathbf{t}=\mathbf{8} \mu$, Porus klein ungeformt,

nicht immer erkennbar ................... Quercus suber $\mathrm{L}$.

\section{DISKUSSION UND SCHLUSSFOLGERUNG}

Die qualitativen Analysen der Honigsproben ergaben, dass von den 11 "Orangenhonigen" nur 3 die Norm erfüllten, um als Orangenhonig zu gelten. Die 
TAB. I. - Zusammenfassung der pflanzlichen und Honigtau-Elemente in 11 spanischen Orangenhonigen.

TABL. I. - Summary of floral elements and honeydew constituents in 11 orange honey samples of Spain.

\section{Abkürzungen Tabelle I.}

Abbreviations Table I.

$1=$ Leitpollen $=$ pollen dominant $=$ predominant pollens.

$\mathrm{b}=$ Begleitpollen $=$ pollen d'accompagnement $=$ secondary pollens.

$\mathrm{e}=$ Einzelpollen $=$ pollen isolé $=$ minor pollens .

Honigtau $=$ indicateur de miellat $=$ honeydew constituents.

$1=$ wenig $=$ peu $=$ low quantity.

$2=$ mittlere Menge $=$ quantité moyenne $=$ mean quantity.

$3=$ viel $=$ grande quantité $=$ high quantity.

$4=$ sehr viel $=$ très grande quantité $=$ very high quantity.

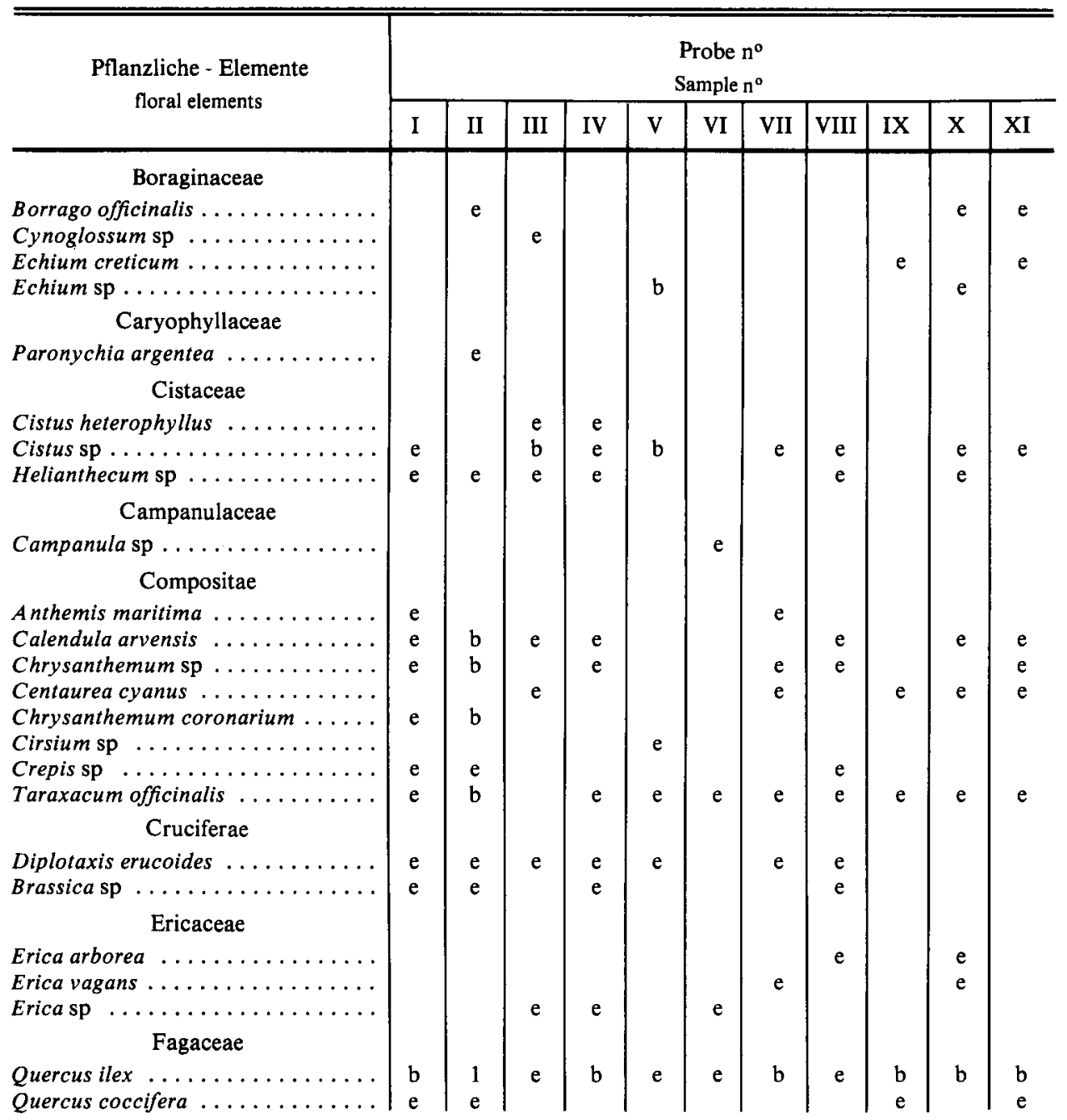




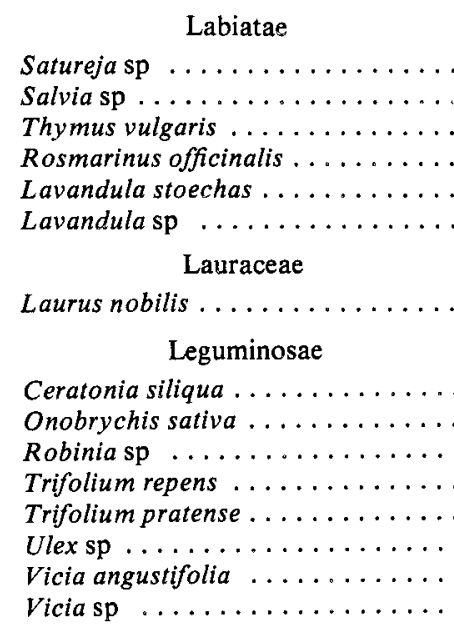

Malvaceae

Lavatera maritima . . . . . . .

Lavatera cretica ..............

Malva silvestris .

\section{Myoporineae}

Myoporum acuminatum

Myrtaceae

Eucalyptus sp

\section{Oleaceae}

Olea europea ...............

Ligustrum $\mathrm{sp}$.

Palmae

Chamaerops sp

Papaveraceae

Hypecoum ............
Plantaginaceae

Plantagn sp . .

\section{Rutaceae}

Citrus sp

\section{Rosaceae}

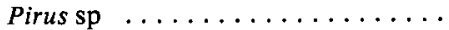

A mygdalus sp

Valerianaceae

Centranthus sp

\section{Vitaceae}

Vitis sp

Parthenocissus sp

Honigtauelemente

honeydew constituents




restlichen 8 Proben zeigten unerwartete Resultate. In den Proben II, IX, und XI (Tab. I) dominieren die anemophilen Pollen von Quercus, welcher durch zwei Arten vertreten sind : Quercus ilex $\mathrm{L}$. und Quercus coccifera L., wobei $Q$. coccifera in der Minderzahl auftritt. In den Proben V, VII und VIII (Tab. I) dominierten die OleaPollen. Sie sind häufig als Begleitpollen in spanischen Honigen anzutreffen (LouvEAux, 1956), öfter zusammen mit Citrus und Eucalyptus. Die Proben III und IV können auf Grund ihrer Zusammensetzung Onobrychis-Olea-Rosmarin als gemischte Honige bezeichnet werden. Das Vorkommen der Onobrychis-Pollen gibt kein Indiz über die Herkunft des Honiges. Diese Proben können trotzdem auf Grund anderer pflanzlicher Elemente (Tab. I), von Bienenstöcken der Huertas aus der Provinz Valencia stammen. Allgemein lässt sich erkennen, dass die Quercus ilex-Pollen in allen 11 Honigproben aus Spanien auftreten. Sie können auf verschiedenem Wege in den Honig gelangen.

$Q$. ilex ist in dieser Region stark verbreitet, wo auch viele Bienenstöcke aufgestellt sind. Nach BUSGEN's Beobachtungen (ZANDER, 1935) werden die Eichenpollen stets sehr reichlich von den Bienen eingetragen. Nach der Blüte liefert die Eiche in manchen Jahren auch viel Honigtau. Das Auftreten der Eichenpollen im Honig wird folgendermassen erklärt (ZANDER, 1935): Die Eiche erzeugt einige Zeit nach der Blüte Honigtau, welcher von den Bienen eingetragen wird. Die am Honigtau klebenden Pollen gelangen so in den Honig. Es könnte aber auch sein, dass die im Honig auftretenden Pollen aus den gespeicherten Pollenvorräten stammen, aus denen sie bei der Verarbeitung durch die Bienen oder durch den Imker beim Schleudern in den Honig gelangen.

Bei meinem Besuch in den Orangenplantagen stellte ich fest, dass ein sehr grosser Teil der Orangenblüten sterile Antheren besitzt, gleichzeitig aber viel Nektar liefert. Im Plantagengebiet sind viele Bienenstöcke aufgestellt. Da die Biene blütenstetig ist, werden die nicht so zahlreichen Citrus-Pollen unter den relativ vielen Bienen aufgeteilt, was zu der Erscheinung der geringen relativen Häufigkeit der im Honig enthaltenen Citrus-Pollen führt. Trotzdem dürfte dieser Honig einen grossen Gehalt an CitrusNektar aufweisen (Orangenblütenaroma), was letzten Endes das Kriterium für den Name dieses Sortenhonigs ist.

In der Praxis jedoch basiert die Beurteilung des Honigs auf rein äusserlichen Merkmalen (Farbe, Viskosität, Aroma und Geschmack). Die bekannten Sortenhonige Lavandula, Thymus, Rosmarinus, Erica, Echium und Cistus werden in Spanien z.B. nur nach ihrem spezifischen Aroma und Geschmack benannt.

\section{DANKSAGUNG}

Dr. A. Maurizıo, Liebefeld (CH), für den Vorschlag des Themas. Den H. H. Prof. Welten und LANG, Herrn Dr. h.c. R. SUTTER, Geobot. Inst. Univ. Bern. Herrn F. ZweILI für die R.E.M. Aufnahmen, Geol. Inst. Bern, Dr. LAvie, Montfavet, Herrn J. TẢrREgA Mor, Castellon für wertvolle Hilfe. 


\section{RÉSUMÉ \\ LA DETERMINATION ET L'ABONDANCE DU POLLEN DE QUERCUS DANS LES MIELS D'ORANGER ESPAGNOLS}

Onze échantillons de miel d'oranger provenant de la Côte espagnole orientale ont fait l'objet d'une étude palynologique. Un pollen inconnu, tricolporé, plus représenté que le pollen de Citrus, est déterminé. Les analyses de miel et les observations de pollen frais en microscopie optique et en microscopie électronique à balayage ( R.E.M. $)$ ), montrent que ce pollen inconnu est un pollen anémophile qui appartient à la famille des Fagaceae, groupe Quercus. Deux espèces sont identifiées dans le miel : Quercus ilex L. et $Q$. coccifera $\mathrm{L}$.

Les résultats d'analyse qualitative dans le miel d'oranger montrent que trois échantillons seulement répondent aux normes pour être classé comme miel d'oranger. Dans tous les échantillons le pollen de Quercus ilex est plus représenté que celui de Citrus. Le parfum de fleurs d'oranger qui caractérise ce miel indique qu'il contient une grande quantité de nectar de fleurs de Citrus.

Les résultats de ce travail montrent la relation existant entre le pourcentage du pollen de Citrus et celui de Quercus. La présence et la prédominance du pollen de $Q$. ilex dans tous les échantillons sont expliquées. Le spectre pollinique trouvé et déterminé dans le miel est présenté dans le tableau I. La morphologie d'espèces de Quercus : Quercus ilex L., $Q$. coccifera L., $Q$. suber L. est décrite. Une clé de détermination est donnée.

\section{SUMMARY \\ IDENTIFICATION AND OCCURRENCE OF QUERCUS-POLLEN IN SOME SPAIN ORANGE HONEYS}

Pollen in 11 orange honey samples from the East coast of Spain were identified. A tricolporate pollen grain which was more frequently observed than citrus pollen has now been identified. Light microscope (" L.M. ") and scanning electron microscope ("R.E.M. ") observations in honey samples and reference pollen grain, show that the unknown, tricolporate pollen grain is anemophil and belongs to Fagaceae, Quercus-group. Two species were identified in honey : Quercus ilex L. and Quercus coccifera L. The qualitative analysis shows that of the 11 honey samples, only 3 can be considered as orange honey. On the whole, in the other 8 samples, $Q$. ilex pollen were more numerous than citrus pollen.

The honey samples had a strong sweet smell of orange blossoms. Therefore, they did contain a large quantity of citrus nectar.

The results show a correlation between the percentage of citrus pollen and Quercus pollen grains. The pollen composition of the honey is shown in Table I. The presence and predominance of Quercus ilex pollen in all honey samples is explained.

The results of the pollenanalyses including a key prepared by L.M. and R.E.M. observations of Quercus spp. from the East coast of Spain : Quercus ilex L., Q. coccifera L., $Q$. suber L. are presented.

\section{LITERATURVERZEICHNIS}

Beug H. J., 1961. - Leitfaden der Pollenbestimmung. Fischer, Stuttgart.

D'Alte J. A. M., 1951. - Anàlise Polinica de Algumas Amostras de Mel, Porto.

DEMIANowicz Z., 1961. - Pollenkoeffizienten als Grundlage der quantitativen Pollen-analyse des Honigs. Pszczelnicze Zeszyty Naukowe, 5, (2), 95-107.

Erdtman G., 1969. - Handbook of Palynology, Munksgaard, Copenhagen. 
ERdTMAN G., 1970. - World pollen flora. Munksgaard, Copenhagen.

EveniUs J., Focke E., 1967. - Mikroskopische Untersuchung des Honigs. In Handbuch der Lebensmittelchemie V, 560-590. Springer, Berlin-Heidelberg-New York.

Hammer O., Jorgensen E. G., Mikkelsen V. M., 1948. - Studies on the content of pollen in Danisch honeysamples. Tidskr. Planteavl, 52, 293-352.

Hyde H. A. and Adams K. F., 1958. - An Atlas of Airborne Pollen Grains, London.

LouveauX J., Vergeron Ph., 1956. - Etude du spectre pollinique de quelques miels Espagnols. Annales de l'A beille, 7, (4), 329-347.

Louveaux J., Maurizio A., Vorwohl G., 1970 b. - Methodik der Melissopalynologie, Apidologie, 1, (2), 193-209.

Maurizio Anna, 1939. - Untersuchungen zur quantitativen Pollenanalyse des Honigs. Mitt. Lebensmitt. unters. Hyg., 30, 27.69.

Maurizıo Anna, 1949 a. - Beiträge zur quantitativen Pollenanalyse des Honigs. 1. Beih. Schweiz. Bienenztg., 2, (18), 320-421.

MAURIzıo Anna, 1955. - Absoluter Gehalt pflanzlicher Bestandteile in Tilia- und Labiatenhonigen. $Z$. Bienenforsch., 3, 32-39.

MaURizio Anna, 1958 a. - Absoluter Gehalt pflanzlicher Bestandteile in Esparsette-, Luzerne-, Orangenund Rapshonigen. Ann. Abeille, 1, 93-106.

Planchais N., 1962. - Le pollen de quelques chênes du domaine méditerranéen occidental. Pollens et Spores, Vol. IV, No 1 .

ZANDER E., 1935. - Beiträge zur Herkunfts-Best. bei Honig, Berlin.

Zander E., MAUrizio Anna, 1975. - Der Honig, Ulmer, Stuttgart.

Woodhouse R. P., 1959. - Pollen grains, New York. 